\title{
Randall H. Morse (ed.): Chromatin remodelling: methods and protocols
}

ISBN: 9781617794766

\author{
Raymond Waters
}

Published online: 6 April 2012

(C) Springer-Verlag 2012

This is an outstanding and timely collection of methods concerning how one can investigate chromatin remodelling. The contributing authors are all at the forefront of this research area. There are detailed laboratory procedures and notes attached to all chapters, and this enable readers to use the volume as a laboratory manual.

The volume contains descriptions of both in vitro and in vivo approaches, and it covers studies with organisms ranging from yeast and plants to mammalian cells. Importantly, it considers both local and global approaches. The latter are emerging as exceptionally powerful tools to rapidly dissect events in chromatin throughout entire genomes. This aspect will have a profound influence on our understanding of how genomes are organised and function, thus impinging on basic and medical sciences to considerable extent. The portion pertaining to global analyses ends with a consideration of how one employs bioinformatics to examine trends in the large datasets from such studies; a crucial topic that is often a bottleneck for many researchers.

The editor, Randall Morse, has to be congratulated on producing such an excellent book; a book that I recommend most highly to researchers in this arena.

R. Waters $(\bowtie)$

Institute of Cancer and Genetics, Medical School,

Cardiff University, Heath Park, Cardiff CF14 4XN, UK

e-mail: watersr1@ cardiff.ac.uk 